\title{
Oriented mentalization-based treatment for borderline personality disorder patients: preliminary results at Camposampiero Mental Health Center
}

\author{
Stefano Carrera, ${ }^{1}$ Guia Pandolfi, ${ }^{1,2}$ Jee Yun Cappelletti, ${ }^{1}$ Walter Padoani, ${ }^{1}$ Silvia Salcuni ${ }^{2}$ \\ ${ }^{1}$ Mental Health Centre, Department of Psychiatry, Camposampiero Hospital, ULSS 6 Euganea, Camposampiero (PD); ${ }^{2}$ Department \\ of Developmental Psychology and Socialisation, University of Padua, Italy
}

\begin{abstract}
This contribution presents two brief reports about preliminary results of 18 months of oriented Mentalization-Based Treatment (MBT), with Borderline Personality Disorder patients, recruited at the Camposampiero Mental Health Center. Following in large part Bateman and Fonagy guidelines for MBT in institutional settings, this paper presents preliminary results in two brief reports related to two cohorts of patient underwent to the oriented MBT in Camposampiero Mental Health Center (MHC). In the first study, we analyzed a group of 9 patients: an anamnestic schedule was administered; then, symptoms (SCL-90-R), psychodiagnostic scale and global health functioning (Health of the Nation Outcome Scale, Structured Clinical Interview for DSM-IV Axis II Disorder [SCID-II], Global Assessment of Functioning, [GAF]), data on service impact and service costs (Cassel Community Adjustment Questionnaire, Patient Evaluation Schedule and folder data) were provided at the beginning $\left(\mathrm{T}_{0}\right)$, at the end of the treatment $\left(\mathrm{T}_{2}\right)$ and 1 year after the end of the MBT project. The second study showed a micro-analytical change on a second patient cohort $(n=6)$ at $T_{0}, 3,6$ and 9 months $\left(T_{1}\right)$ were presented considering specifically mentalization (Comparative Psychotherapy Process Scale, Modes of Mentalization Scale, Mentalization Imbalances Scale) and patienttherapist session evaluation trends (Session Evaluation Questionnaire) and patient reflective functioning at $\mathrm{T}_{0}$ and $\mathrm{T}_{2}$ (Reflective Functioning Questionnaire). Aims and hypotheses of the two studies pointed out how the oriented MBT bring to an improvement of the overall functioning of the patients, a reduction of the symptoms, a decrease of the diagnostic criteria for the Borderline Personality Disorder (BPD) and the other Axis II disorders, a reduction of the workload hours for the MHC staff and of the costs of the assistance. The analyses of both the studies were carried out using non-parametric statistics (Friedman test, Spearman correlation, Chi-square). Preliminary results confirmed the improvement in the overall functioning of patients (GAF), the reduction in BPD-related symptoms and in diagnostic criteria for BPD (SCID-II), the improvement of patients' mentalization skills, and a significant reduction in workload for health staff. Standing the limits and the preliminary results, the two brief reports demonstrate the feasibility of an oriented MBT within an Italian Public Service and the effectiveness of this treatment pathway for patients with BPD, leaving some open questions to stimulate a fruitful clinical discussion.

Correspondence: Stefano Carrera, Mental Health Center, DepartCamposampiero (PD), Italy.

E-mail: stefano.carrera@studenti.unipd.it
\end{abstract}

Citation: Carrera, S., Pandolfi, G., Cappelletti, Y. E., Padoani, W., \& Salcuni, S. (2018). Oriented mentalization-based treatment for borderline personality disorder patients: preliminary results at Camposampiero Mental Health Centre. Research in Psychotherapy: Psychopathology, Process and Outcome, 21(3), 178-189. doi: 10.4081/ripppo. 2018.336

Contributions: the authors contributed equally.

Conflict of interest: the authors declare no potential conflict of interest.

Funding: none.

Received for publication: 17 September 2018.

Revision received: 28 November 2018.

Accepted for publication: 5 December 2018.

This work is licensed under a Creative Commons Attribution NonCommercial 4.0 License (CC BY-NC 4.0).

CCopyright S. Carrera et al., 2018

Licensee PAGEPress, Italy

Research in Psychotherapy:

Psychopathology, Process and Outcome 2018; 21:178-189

doi:10.4081/ripppo.2018.336
Key words: Mentalization-based treatment; Borderline personality disorder; Symptoms; Mental Health Center.

\section{Introduction}

Severe Personality Disorders (PDs) represent clinical conditions of increasing impact on Mental Health Services: European studies (Lora, 2009; Sanza, Asioli, \& Ferrannini, 2010; Torgensen, Kringlen, \& Cramer, 2001) show that the prevalence of PDs in the general population is of about $13 \%$. Between them, European research estimate a prevalence of Borderline Personality Disorder (BPD) between $0.7 \%$ and $1.8 \%$ of the general population (Chapman \& Fleisher, 2017; Torgensen et al., 2001), and between $15 \%$ and $25 \%$ in the clinical population (Chapman \& Fleisher, 2017; Gunderson \& Links, 2014).

The DSM-5 (APA, 2013) describes BPD as a pervasive pattern of instability of interpersonal relationships, self-image and mood, and a marked impulsivity, which begins in early adulthood and is present in various con- 
texts, and defines its critical issues in interpersonal relationships and global functioning, identity disturbance and emotional dysregulation. At the same time, it underlines the high comorbidity of PDs with Axis I conditions, with particular reference to Mood Disorders, Substance Use Disorders and Eating Disorders.

Following these considerations, it is clear how, especially in Public Health Services, it is important to have therapeutic pathways able to deal (in an effective and efficient way) with the pressing request for treatment from such a complex clinical population.

In 2012, the staff of the Department of Psychiatry of Camposampiero Hospital decided to structure a specific therapeutic pathway for patients with BPD, which constitute about $13 \%$ of primary diagnoses. A specialized team was set up and, over the course of the following two years of reflection, training and theoretical-clinical analysis, reviewed the national and international literature about the topic and finally opted for the Mentalization-Based Treatment (MBT) model (Bateman \& Fonagy, 2010a, 2010b; Fonagy, Target, \& Bateman, 2018). The choice of this protocol was dictated by the evidence that the theoretical approach and the method seemed to respond, better than others, to the care direction identified by the team, to the patients' clinical needs and to the resources available at the Service, which made it possibile to apply it in an Italian Public Health Service.

Mentalizing is defined as an "imaginative mental activity, namely, perceiving and interpreting human behavior in terms of intentional mental states (e.g., needs, desires, feelings, beliefs, goals, and reasons)" (Fonagy, Gergerly, \& Target, 2007, p. 288). Lately, Fonagy et al. have broadened their concept of mentalization, by introducing four opposite polarities that re-defined mentalization as a "dynamic capacity that is influenced by stress and arousal, particularly in the context of specific attachment relationships" (Fonagy, Bateman \& Luyten, 2012, p. 19). Mentalization opposite polarities represent a "balanced systems where a dysfunction at one pole may manifest as the unwarranted dominance of the opposite polarity" (Fonagy \& Allison, 2011, p. 106): i) affective $v s$ cognitive mentalization, ii) controlled (explicit) $v s$ automatic (implicit) mentalization, iii) other-oriented $v s$ selforiented mentalization, and iv) externally focused $v s$ internally focused mentalization. Patients with BPD are characterized by the presence of severe deficits in mentalization capacity which leads to concrete pre-mentalizing way of thinking; their concrete thinking is characterized by the equation of internal and external world, called psychic equivalence; a use of pretend mode/pseudologic way of thinking in which thoughts and feelings can be envisioned and talked about, but they correspond to nothing real; and finally, a teleological mode of thinking that appears when some intentions is imputed from what is just physically apparent (Fonagy \& Allison, 2011). BP patients consequently presented problems in emotional regulation and in managing impulsivity, especially in the context of interpersonal relationships (Fonagy et al., 2018).

Standing these difficulties, Bateman and Fonagy (2013a) developed a time-limited Mentalization Based Treatment (MBT) which structure interventions that promote the development of mentalizing process and capability. It is provided within 18 months and is articulated in a double setting path (individual and group setting), both with weekly sessions. Individual sessions are 50 minutes long, while group sessions last 90 minutes. The path can be structured as a day-hospital or as an intensive outpatient treatment. The main purposes of MBT are to increase mentalization capacity by maintaining a focus on the mind of the patient, the therapist and other people, and to improve patients' ability to manage the emotional states (Fonagy et al., 2018). MBT has undergone several efficacy tests, particularly in Anglo-Saxon countries and Northern Europe, where this treatment approach is now considered an evidence-based treatment (APA, 2005) in clinical practice for patients with BPD (David, Lynn, \& Montgomery, 2018; see also Madeddu et al.'s meta-analytic work, 2012).

MBT has positive outcomes in relation to BPD diagnosis, symptoms associated with the disorder, social and interpersonal functioning, personality pathology and overall functioning (Bateman \& Fonagy, 2008b; Fonagy et al., 2018; Jorgensen \& Kjolbye, 2007), moreover it is associated with a reduced costs of the assistance due a reduction in suicidal attempts, self-harming behaviors and requests for medical cares (Bales et al., 2012; Madeddu, Aquaro, $\&$ Preti, 2012). These changes are maintained over time, during the follow-up period (Bales et al., 2012; Bateman \& Fonagy, 2013a, 2013b).

To conclude, MBT shows a good effectiveness for the treatment of patients with severe self-injurious and anticonservative behaviors, and provides improvements both in the short and long term, whether performed in a dayhospital or in an intensive outpatient setting. To our knowledge, only few authors have investigated the efficacy and effectiveness (intervention studies can be placed on a continuum, with a progression from efficacy trials to effectiveness trials. Efficacy can be defined as the performance of an intervention under ideal and controlled circumstances, whereas effectiveness refers to its performance under real-world conditions) of MBT for patients with BPD in the Italian panorama. Recently, a research group led by Dr. Bergonzini (National Hospital SS. Antonio and Biagio and Cesare Arrigo of Alessandria, Italy), in collaboration with the Department of Psychology of the University of Turin, has conducted an exploratory RCT with MBT vs TAU for the evaluation of comparative effectiveness between treatments, on 5 patients (Bergonzini et al., 2013). Apart from the research by Bergonzini et al., no other studies related to the effectiveness of MBT in the Italian panorama have been published 
to date. This evidence, if on one hand highlights the difficulty of Italian Public Services in integrating clinical with research practice, on the other hand, informs about the novelty represented by this form of treatment and the need to conduct further research on its effectiveness in different clinical contexts.

While the mentalization construct was delineating, Fonagy developed a specific measure, first defined as Reflective Self-Function and subsequently Reflective Functioning Scale. It was a scale applicable to the transcripts of numerous clinical interviews which has been applied in some efficacy studies related to other psychotherapeutic forms (Levy, Meehan, \& Kelly, 2006) and in research for the evaluation of attachment (Fonagy, Steele, Steele, Moran, \& Higgit, 1991; Slade, Grienenberger, Bernbach, Levy, \& Locker, 2005), paradoxically only recently was used in MBT efficacy studies (Fonagy et al., 2018). Concerning the evaluation of the effectiveness of MBT, the meta-analysis of Madeddu et al. (2012) concludes stating that to date there are no empirical studies that demonstrate the effective increase in mentalization capacity in patients undergoing treatment, which is the real objective of MBT, although the evidence for their considerable clinical improvement is abundant.

The illustrated evidence, also considering the limits of the research conducted so far, led the American Psychological Association (Division 12) to consider the Mentalization Treatment for BPD a psychotherapy of only probable efficacy and effectiveness in 2005, and definitively as an Evidence Based Treatment recently (David, Lynn, \& Montgomery, 2018; Fonagy et al., 2018; Morandotti et al., 2018). However, further studies are expected to include measures of mentalization capacity to confirm the effectiveness of the MBT.

At Camposampiero Mentalization Imbalances Scale (MHC), the oriented MBT path started in April 2014; it was structured on the basis of Fonagy and Bateman's model (Bateman \& Fonagy, 2010a), adapted to the contingent needs of the specific context of Camposampiero MHC. Before starting the path, patients followed a psycho-educational program that included 12 sessions distributed over a period of about three months. The oriented MBT program included an intensive outpatient treatment of 18 months, during which each patient participated in a double (individual and group) setting treatment, both on a weekly basis. Consistent with what was suggested by the authors (Bateman \& Fonagy, 2010a), the two settings must be carried out contemporarily during the 18 months of treatment, and they both are composed of about 45 sessions each. The group, which can be placed within the category of semiopen groups, consists of a maximum of 9 members. The clinical team was composed of 3 psychiatrists, 1 clinical psychologist, 3 nurses, and 7 trainees in psychotherapy; the 3 team leaders attended the MBT training held by Fonagy and Bateman. Each trainee in psychotherapy as well as the psychiatrists and the clinical psychologist, followed MBT guidelines in treating one or two patients in the individual psychotherapies path. The team met weekly so that the team leaders could provide supervision to trainees' individual psychotherapies, by reading and discussing the sessions reports and/or the audio-recorded tape. Moreover, the team discusses the progress of the psychotherapies, evaluates the possibility of including other patients in the program, discusses the answers provided by patients at the afore mentioned tests and, last but not least, keeps the équipe updated on BPD and MBT. A silent observer (an in-training psychologist) participated at the groups with the only task of transcribing what happens in the session and writing up the summary reports. Individual psychotherapies, composed of about 45 sessions each, were conducted by the remaining psychiatrists and psychologists (with the exclusion of the group leader) and by the trainees in psychotherapy. Individual and group psychotherapy sessions were constantly audio recorded, and supervisions were provided on these audio records, in contrast with MBT standard protocol in which supervision is based on video-recorded sessions (Fonagy et al., 2018).

The oriented MBT path at the MHC of Camposampiero presented some peculiarities that integrate the original model of Fonagy and Bateman (2010) with the practice of Good Psychiatric Management by Gunderson and Links (2014). In particular, a nurse had an active role in the co-management of the group as co-conductor: the decision to involve an MHC nurse as a conductor of the MBT group allowed the patients to have a continuity between the group and the daily life of the MHC. Moreover, the psychiatrist of the MHC interacts with the MBT team to establish whether a patient should be included in the program and remains the case manager of the patient during the entire path, in order to ensure a fundamental continuity of care (the MHC psychiatrist performs a first job of diagnosis and evaluation of the MBT project appropriateness for that specific patient and fixes periodic meetings with him, during which he discusses with him the trend of the path, supports and increases its motivation to participate and accompanies it in the phases of the conclusion of the course and in those following the end).

In Camposampiero MHC, no more than one oriented MBT program and related research is unfolding at each time. In agreement with the MHC psychiatrist and the psychotherapist responsible for the individual psychotherapy, the patient can decide whether or not to take part to the oriented MBT path and must sign an Informed Consent (Participants declared and signed written informed consent in line with the current regulations laws D. Lgs 196/2003 and EU GDPR 679/2016 on data protection e to consent to the processing and communication of data personal, within the limits, for the purposes and for the duration specified by current laws - Legislative Decree 196/2003 and EU GDPR 679/2016. Manager of the research undertakes to fulfill the obligations established by the current legislation in terms 
of collection, treatment and conservation of sensitive data. Similarly, the individual therapist and the group conductor were also invited to sign the release).

\section{Aim and hypothesis}

Since the beginning of the oriented MBT project, in October 2014, the Camposampiero team has set up a research protocol to evaluate the effectiveness of treatment and its impact on the costs of the assistance. We expected that MBT would be associated, with a recognized validity in determining a statistically significant change, to an improvement of the BPD-associated symptoms, of mentalization capacity, of emotional regulation (above all of auto- and hetero-directed rage and aggression), of prevention and management of self-injurious and anti-conservative behaviors, and of the quality of interpersonal relationships; these changes would allow a reduction of both the workload for the staff of the MHC, in particular for the MHC personnel, and the costs of assistance in terms of decrease in the number of hospitalizations, emergency direct access and prescribed drugs.

Our paper was mainly based on a wide research question and its consequent implication: is it possible to implement an oriented MBT care path, as articulated by Fonagy and Bateman, in an Italian Public Service? Standing the ongoing therapy of the present research project, we will present two studies or brief reports, showing the first results of our attempt to manage an oriented MBT program in an Italian public health care service.

\section{Study 1}

Research questions: i) Is it possible to apply oriented MBT program into an Italian Public Mental Health Center? ii) Can an oriented MBT care path provide a statistically significant change in relation to BPD-associated symptoms, emotional regulation (above all of self- and hetero-directed rage and aggression), prevention and management of selfinjurious and anti-conservative behaviors, improve the quality of interpersonal relationships and reduce the workload for the Service staff and the costs of assistance?

\section{Materials and Methods}

\section{Participants}

On a 9 patients group that had completed the oriented MBT protocol, symptoms (SCL-90-R), psychodiagnostic scale and global health functioning (Health of the Nation Outcome Scale [HoNOS], Structured Clinical Interview for DSM-IV Axis II Disorder [SCID-II], Global Assessment of Functioning [GAF]), data on service impact and service costs (PES, folder data) were compared at the beginning of the treatment $\left(\mathrm{T}_{0}\right)$, at the end of the treatment $\left(\mathrm{T}_{2}\right)$ and 1 year (FU1) after the end of the oriented MBT project. This first group $(\mathrm{N}=9)$ was composed by $23 \%$ males and $77 \%$ females: consistently with the literature on the frequency of BPD in the two sexes (Gabbard, 2015), with a female-male proportion of $3: 1$ (Chapman \& Fleisher, 2017). About the males, $50 \%$ of them were unoccupied, while $50 \%$ were businessmen; $50 \%$ were married, while $50 \%$ were unmarried and all of them were high school graduated; their average Socio-Economical Status (Hollingshead, 1975) was medium. About the females, $29 \%$ of them were unoccupied, while $71 \%$ were employed; $43 \%$ were married, while $57 \%$ were unmarried and $86 \%$ of them were high school graduated, while $14 \%$ had a third level degree; their average Socio-Economical Status (Hollingshead, 1975) was medium.

\section{Procedure}

According to the international literature, the research protocol was assessed following these steps:

i) Time $0\left(\mathrm{~T}_{0}\right)$ : pre-treatment screening

ii) Time $1\left(T_{1}\right): 9$ months from the beginning of the oriented MBT project.

iii) Time $2\left(\mathrm{~T}_{2}\right)$ : at the end of the oriented MBT project (18th month).

iv) Follow-up (FU): one year after the end of treatment.

\section{Measures}

Psychodiagnostic and global functioning tools (administered at $\mathrm{T}_{0}, \mathrm{~T}_{2}$ and $\mathrm{FU}$ ) are both self- and clinician-report tools. The self-report tools (SCID-II Questionnaire, SCL90R and Cassel Community Adjustment Questionnaire, CAQ) are delivered to the patients by the MBT team and withdrawn after completing them. Tow clinician-report tools (GAF and HoNOS) are completed by the therapist of each patient, in collaboration with his or her MHC psychiatrist. The SCID-II Interview is provided to the patients by a trainee in psychotherapy who is a member of the MBT team but doesn't have a role of psychotherapist in the oriented MBT path. The same trainee collects folder data to complete the PES.

SCID-II (First \& Gibbon, 2004) was administered according to the diagnostic criteria foreseen by the DSMIV-TR (APA, 2000). The SCID-II consists of 13 subscales, each corresponding to a Personality Disorder described in the DSM-IV-TR: Avoidant, Dependent, Obsessive-compulsive, Passive-aggressive, Depressive, Paranoid, Schizotypical, Schizoid, Histrionic, Narcissistic, Borderline, Antisocial and Not Otherwise Specified. The scoring is done by counting the number of items previously coded 3. On the scoring board is indicated the cutoff for each disorder, so that it is possibile to diagnose a personality disorder if the number of items coded as 3 exceeded the cut-off expected for that disorder.

GAF (Jones, Thornicroft, Coffey, \& Dunn, 1995) is a clinician-report scale that assesses the patient's psychological functioning on a continuous from 0 to 100 , where 0 corresponds to the lowest degree of functioning, while 100 corresponds to an optimal global level of functioning. 
The GAF presents 10 anchor points, each corresponding to a description of the expected functioning of the examined patient: the clinician must choose the description that is closest to the specific person under evaluation, with the possibility to using an intermediate score among those proposed by the GAF.

Symptom Check List-90-Revised (SCL-90R; Derogatis, 1994) is a self-report psychiatric symptomatic scale, based on a 5 -point Likert scale $(0=$ Not at all, $1=$ Little, $2=$ Moderately, $3=$ Very, $4=$ Very much), in which the patient is asked to indicate how intense $\mathrm{s} /$ he has suffered from the problems described in each of the 90 items. The scoring is based on 9 subscales (Somatization, Obsessivecompulsive, Interpersonal Sensitivity, Depression, Anxiety, Hostility, Phobias, Paranoia, Psychoticism) and a global index (Global Score Index, GSI) of symptomatic severity overall. The cut-off for each subscale and for the GSI is equal to 1 .

CAQ is a self-administered questionnaire, specifically designed to evaluate the patient's behaviors year prior to the evaluation; it consists of 12 items that collect the details of self-harming and hetero-aggressive behaviors, substance abuse, suicide attempts, number and duration of hospitalizations, and the working and living situation of the subject. This scale permits the assessment of the progresses of individual items over time, on the base of non-descriptive statistics.

HoNOS (Lora et al., 2001; Wing et al., 1998) is a clinician-report scale specifically designed for the evaluation of mental treatment results for psychiatric patients in charge to the Services. It consists of 12 items, based on 4 dimensions: psychiatric experiences (e.g. anxiety, depression, hallucinations), basic functions deficiency (e.g. psychomotor retardation, cognitive impairments), behavioral problems (e.g. auto- and hetero-direct violence) and problems in functional autonomy (residential, occupational, economic, interpersonal and social autonomy). The clinician rates each item on a 5 -point Likert scale $(0=$ No problem; $1=$ Small problem; $2=$ Small problem; $3=$ Medium problem; $4=$ Serious or very serious problem). By adding the scores of each item, clinicians obtain a total score (between 0 and 48) which describes the patient's overall impairment. It is also possible to compare the trend of single items or dimensions at different times.

Patient Evaluation Schedule (PES) is a specific schedule for the collection of patient's folder-data, designed by the Camposampiero MHC team. The schedule is meant to obtain data on the patient's history in terms of clinical data (e.g. co-morbidities with other Personality Disorders and other Major Psychiatric Disorders, the presence of self-injurious acts, episodes of suicide attempts, substance and alcohol abuse, etc.) and information related to the impact on the Service (e.g. number of emergency access and days of psychiatric admission, number of individual and group therapy sessions, psychotropic drugs prescription, discharge or management at the end of the project and at the FU, etc.).

\section{Data analysis}

Samples description was provided. Symptoms self-reported (SCL-90R), psycho-diagnostic scale and global health functioning (HoNOS, GAF, SCID-II) reported by the clinicians, and data on service impact and service costs (CAQ, PES and folder data) were compared on a 9 patients sample, at the beginning of the treatment $\left(\mathrm{T}_{0}\right)$, at the end of the treatment $\left(\mathrm{T}_{2}\right)$ and 1 year $(\mathrm{FU})$ after the end of the oriented MBT project, using non-parametrical repeated measures analysis (Friedman test).

\section{Results}

Before starting with the oriented MBT program, the 9 patients presented a quite critical clinical profile, characterized, in particular, by a positive SCID-II Borderline Personality Disorder Scale and the presence of Hostility, Depression, Obsessive-Compulsive, Sleep problems, and Anxiety symptoms in the SCL-90R (Tables 1 and 2). The SCL-90R General Symptom Index rated by the pa-

Table 1. Timeline tools administration oriented Mentalization-Based Treatment (MBT) protocol.

\begin{tabular}{|c|c|c|c|c|c|c|}
\hline & $T_{0}-$ Start & $3 \mathrm{~ms}$ & $6 \mathrm{~ms}$ & $\mathrm{~T}_{1}-9 \mathrm{~ms}$ & $\mathrm{~T}_{2}-\mathbf{- 1 8} \mathrm{ms}$ & FU - 1 yr \\
\hline $\begin{array}{l}\text { Study } 1 \text { : } \\
N=9 \text { patients }\end{array}$ & $\begin{array}{c}\text { SCL-90R } \\
\text { SCID-II } \\
\text { HoNOS } \\
\text { GAF } \\
\text { CAQ }\end{array}$ & - & - & - & $\begin{array}{c}\text { SCL-90R } \\
\text { SCID-II } \\
\text { HoNOS } \\
\text { GAF } \\
\text { CAQ }\end{array}$ & $\begin{array}{c}\text { SCL-90R } \\
\text { SCID-II } \\
\text { HoNOS } \\
\text { GAF } \\
\text { CAQ }\end{array}$ \\
\hline $\begin{array}{l}\text { Study } 2 \text { : } \\
\mathrm{N}=6 \text { patients } \\
\text { (ongoing oriented } \\
\text { MBT program) }\end{array}$ & $\begin{array}{c}\text { SEQ-P } \\
\text { SEQ-T } \\
\text { CPPS } \\
\text { MMS } \\
\text { MIS } \\
\text { RFQ }\end{array}$ & $\begin{array}{c}\text { SEQ-P } \\
\text { SEQ-T } \\
\text { CPPS } \\
\text { MMS } \\
\text { MIS }\end{array}$ & $\begin{array}{c}\text { SEQ-P } \\
\text { SEQ-T } \\
\text { CPPS } \\
\text { MMS } \\
\text { MIS }\end{array}$ & $\begin{array}{c}\text { SEQ-P } \\
\text { SEQ-T } \\
\text { CPPS } \\
\text { MMS } \\
\text { MIS } \\
\text { RFQ }\end{array}$ & - & - \\
\hline
\end{tabular}

SCL-90R, Symptom Check List-90-Revised; SCID-II, Structured Clinical Interview for DSM-IV Axis II Disorder; GAF, Global Assessment of Functioning; CAQ, Cassel Community Adjustment Questionnaire; HoNOS, Health of the Nation Outcome Scale; SEQ-T and SEQ-P, Session Evaluation Questionnaire-Therapist version and -Patient version; CPPS, Comparative Psychotherapy Process Scale; MMS, Modes of Mentalization Scale; MIS, Mentalization Imbalance Scale; RFQ, Reflective Functioning Questionnaire. 
tient, as well as the Global Assessment Functioning evaluated by the clinicians in $\mathrm{T}_{0}$, enlightened a compromised level of functioning, indicating that the patients had Moderate symptoms (e.g., flat affect and circumlocutory speech, occasional panic attacks) and moderate difficulty in social, occupational, or school functioning (e.g., few friends, conflicts with peers or co-workers). The HoNOS scale rated by clinicians indicated a moderate patients' impairment.

Each patient received in the 18 months period from $\mathrm{T}_{0}$ to $T_{2}$ a total of 45 individual oriented MBT psychotherapy sessions and 45 group oriented MBT psychotherapy sessions. In $\mathrm{T}_{2}$ and at the $\mathrm{FU}$ none of the variables positive in $\mathrm{T}_{0}$ from a clinical point of view remained critical considering the clinical cut-off. We analyzed the time-trend of the variables and found that MBT effect seems to affect the change between $T_{0}$ and $T_{2}$, which remains constant after one year. At the end of the treatment and at the follow up after one year, both the SLC-90R self-report and the HoNOS, SCID-II and GAF clinician-reports showed the absence of personality disorders, and of heavy psychiatric symptoms, attesting a 71-80 level of functioning, in which: If symptoms are present, they are transient and expectable reactions to psychosocial stressors (e.g., difficulty concentrating after family argument); no more than slight impairment in social, occupational, or school functioning (e.g., temporarily falling behind in schoolwork.

With respect to clinical variables related to patients' hospitalizations, clinical folders show that regular MHC visits remained quite stable along the measured period $\left(\mathrm{T}_{0}: \mathrm{M}=9,33, \mathrm{sd}=6,30 ; \mathrm{T}_{2}: \mathrm{M}=10, \mathrm{sd}=, 50 ; \mathrm{FU}: \mathrm{M}=6,44\right.$, $\mathrm{sd}=8,00)$. Patients' days of hospitalization due to a critical psychosocial and symptomatic situation, decreased significantly from a mean of $5,78(\mathrm{sd}=13,43)$ in $\mathrm{T}_{0}$, before the treatment, to a mean of 0 , both in $\mathrm{T}_{2}$ and after 1-year follow up; urgent hospital accesses as well the use of different kind of substance or medications, decreased significantly from $\mathrm{T}_{0}$ to $\mathrm{FU}$ showing an improved adjustment from a clinical point of view, with a reduction in workload for Service staff and costs of assistance (Table 3).

Among the self-report tools, in the research design, there is also the administration of the CAQ useful for evaluating the trend over time of some maladaptive behavior typical of Borderline subjects. As shown in

Table 2. Comparison of repeated measures Generalized Linear Model (GLM) at the beginning $\left(T_{0}\right)$, the end $\left(T_{2}\right)$ and after one year follow up ( $\mathrm{FU})(\mathrm{N}=9$ patients).

\begin{tabular}{|c|c|c|c|c|c|c|c|c|c|}
\hline & \multirow[b]{2}{*}{ Clinical Scales* } & \multicolumn{2}{|c|}{$\mathbf{T}_{\mathbf{0}}$} & \multicolumn{2}{|c|}{$\mathbf{T}_{2}$} & \multicolumn{2}{|c|}{ FU 1 year } & \multirow[b]{2}{*}{$\begin{array}{c}\text { Friedman test } \\
* \mathbf{P}<.05 \\
* * \mathbf{P}<.001\end{array}$} & \multirow[b]{2}{*}{ Post hoc } \\
\hline & & $\mathbf{M}$ & sd & M & sd & $\mathbf{M}$ & sd & & \\
\hline \multirow{2}{*}{$\begin{array}{l}\text { Health of } \\
\text { Nations } \\
\text { Outcome } \\
\text { Scale } \\
\text { DSM-IV }\end{array}$} & HoNOS & 14,9 & 4,3 & 7,6 & 2,8 & 9,8 & 10,1 & $*$ & $\mathrm{~T}_{0}>\mathrm{T}_{2}=\mathrm{FU}$ \\
\hline & $\begin{array}{l}\text { Global Assessment } \\
\text { Functioning }(<70)\end{array}$ & $53,8 * * *$ & 18,4 & 75,0 & 14,4 & 77,3 & 13,6 & $*$ & $\mathrm{~T}_{0}>\mathrm{T}_{2}=\mathrm{FU}$ \\
\hline \multirow{12}{*}{$\begin{array}{l}\text { SCID-II } \\
\text { Personality } \\
\text { Disorder } \\
\text { Scales }\end{array}$} & Bordeline $(>5)$ & $5,4 * * *$ & 2,1 & 3,2 & 2,6 & 1,3 & 1,9 & $* *$ & $\mathrm{~T}_{0}>\mathrm{T}_{2}>\mathrm{FU}$ \\
\hline & Depression $(>5)$ & 3,2 & 1,4 & 0,9 & 1,2 & 1,6 & 1,9 & .077 & $\mathrm{~T}_{0}>\mathrm{FU}>\mathrm{T}_{2}$ \\
\hline & $\mathrm{PA}(>4)$ & $3,0 * * *$ & 1,9 & 1,6 & 1,5 & 1,7 & 1,5 & $*$ & $\mathrm{~T}_{0}>\mathrm{T}_{2}=\mathrm{FU}$ \\
\hline & Paranoid $(>4)$ & 2,6 & 2,1 & 1,9 & 1,3 & 1,3 & 1,3 & .053 & \\
\hline & Obsessive Compulsive $(>4)$ & 1,8 & 1,2 & 1,4 & 1,2 & 1,2 & 1,2 & n.s. & \\
\hline & Avoidant $(>4)$ & $1,7 * * *$ & 1,5 & 0,9 & 0,9 & 0,7 & 1,3 & $*$ & $\mathrm{~T}_{0}>\mathrm{T}_{2}=\mathrm{FU}$ \\
\hline & Dependent $(>5)$ & 1,6 & 1,7 & 0,8 & 1,1 & 0,6 & 0,7 & n.s. & \\
\hline & Istrionic $(>5)$ & 1,6 & 1,7 & 1,4 & 1,7 & 0,9 & 1,7 & n.s. & \\
\hline & $\operatorname{SCT}(>5)$ & 1,4 & 1,3 & 0,8 & 1,4 & 0,7 & 0,9 & $*$ & $\mathrm{~T}_{0}>\mathrm{T}_{2}=\mathrm{FU}$ \\
\hline & Antisocial (>3) & 1,2 & 2,3 & 1,0 & 1,5 & 0,4 & 1,3 & n.s. & \\
\hline & Narcissistic $(>5)$ & 1,1 & 1,4 & 1,2 & 1,2 & 0,8 & 0,7 & n.s. & \\
\hline & $\operatorname{SCD}(>4)$ & 0,9 & 1,4 & 0,8 & 1,0 & 0,3 & 1,0 & n.s. & \\
\hline \multirow{11}{*}{$\begin{array}{l}\text { SCL-90R } \\
\text { Symptomatic } \\
\text { Scales } \\
(>1)\end{array}$} & Hostility & $2,0 * * *$ & 0,4 & 0,9 & 0,3 & 0,7 & 0,2 & $*$ & $\mathrm{~T}_{0}>\mathrm{T}_{2}=\mathrm{FU}$ \\
\hline & Depression & $1,5 * * *$ & 0,3 & 0,9 & 0,3 & 0,7 & 0,2 & $*$ & $\mathrm{~T}_{0}=\mathrm{T}_{2}>\mathrm{FU}$ \\
\hline & Obsessive-Compulsive & $1,5 * * *$ & 0,6 & 0,9 & 0,8 & 0,6 & 0,6 & $*$ & $\mathrm{~T}_{0}=\mathrm{T}_{2}>\mathrm{FU}$ \\
\hline & Sleep problems & $1,5 * * *$ & 0,5 & 0,5 & 0,2 & 0,6 & 0,2 & n.s. & \\
\hline & Paranoid & $1,4 * * *$ & 0,3 & 0,7 & 0,2 & 0,5 & 0,2 & $*$ & $\mathrm{~T}_{0}>\mathrm{T}_{2}=\mathrm{FU}$ \\
\hline & Interpersonal Sensitivity & $1,2 * * *$ & 0,2 & 0,6 & 0,2 & 0,5 & 0,2 & $*$ & $\mathrm{~T}_{0}>\mathrm{T}_{2}=\mathrm{FU}$ \\
\hline & Anxiety & $1,2 * * *$ & 0,4 & 0,6 & 0,7 & 0,5 & 0,4 & $*$ & $\mathrm{~T}_{0}>\mathrm{T}_{2}=\mathrm{FU}$ \\
\hline & Psychoticism & 0,9 & 0,5 & 0,3 & 0,3 & 0,2 & 0,2 & .050 & $\mathrm{~T}_{0}>\mathrm{T}_{2}=\mathrm{FU}$ \\
\hline & Somatization & 0,8 & 0,5 & 0,9 & 0,7 & 0,8 & 0,5 & n.s. & \\
\hline & Phobia & 0,6 & 0,2 & 0,3 & 0,2 & 0,3 & 0,1 & n.s. & \\
\hline & General Symptoms Index & $1,3 * * *$ & 0,1 & 0,8 & 0,2 & 0,7 & 0,1 & $*$ & $\mathrm{~T}_{0}>\mathrm{T}_{2}=\mathrm{FU}$ \\
\hline
\end{tabular}

For each clinical scale we considered the manualized cut-off of pathological functioning, in between parentheses and $* * *$ indicated values above the clinical cutoff; $* \mathrm{P}<.05 ; * * \mathrm{P}<.01$. 
Table 3, during the 18 months of treatment $\left(\mathrm{T}_{2}\right)$ the selfinjurious behaviors, the suicide attempts, the auto and hetero-direct aggressiveness showed a substantial decrease that persisted also in the 12 months following the conclusion of the project (FU).

These outcomes are also supported by data emerging from PES and data folder, which show a clear reduction of self-harming behaviors over the three evaluation times. Particularly interesting is the data relating to the progressive decrease, with time, of the number and frequency of urgent accesses, hospital admissions and psychiatric treatments provided. This result also points out to a lower use of drugs, especially antipsychotics, that reiterates the improvement of global functioning and allows us to make a general reflection on the impact that oriented MBT treatment has on the Health Service. Obviously, the improvements observed in the sample of the 9 patients correspond to positive consequences in terms of reduction of the commitment required to the staff of the $\mathrm{MHC}$ and reduction of the health costs to which the affected structure is subjected. Regarding the frequencies related to the employment status and the housing situation of the patients, it emerged that the changes obtained during the treatment were maintained also during the follow-up period.

\section{Study 2}

Research questions: i) Does the MBT lead to an increase in the mentalizing capacity of patients? More specifically, will patients present a reduction in imbalances of mentalization and in pre-mentalizing modes? ii) Are these modifications accompanied to some other dimensions of psychotherapy process?

\section{Materials and Methods}

\section{Participants}

Due to the fact the MBT is an open-group, we started to analyze the micro-analytical mentalization changes and session feature trends and reflective functioning at $\mathrm{T}_{0}$ and $\mathrm{T}_{1}$ (by using the Reflective Functioning Questionnaire, RFQ), on a new group of 6 patients, composed by 2 males and 4 females. About the males, 50\% were unoccupied,

Table 3. Percentage of change in the comparison of Cassel Community Adjustment Questionnaire (CAQ) items and Patient Evaluation Schedule (PES) variables frequencies at the beginning $\left(T_{0}\right)$, the end $\left(T_{2}\right)$ and after one year follow up (FU) (N=9 patients).

\begin{tabular}{|c|c|c|c|c|}
\hline PES & $\begin{array}{l}\mathrm{T}_{0} \\
\%\end{array}$ & $\begin{array}{l}\mathrm{T}_{2} \\
\%\end{array}$ & $\begin{array}{c}\text { FU } \\
\%\end{array}$ & $\%$ Clinical Change \\
\hline Urgent Hospital Access & 23 & 8 & 8 & $* *$ \\
\hline Substance Abuse & 15 & 15 & 8 & $* * *$ \\
\hline Alcohol Abuse & 31 & 23 & 15 & $* *$ \\
\hline Self-Harming Behavior & 23 & 8 & 8 & $* *$ \\
\hline Suicide Attempt & 8 & 8 & 0 & $* * * *$ \\
\hline Anxiety Medicine Use & 46 & 15 & 23 & $* *$ \\
\hline Depression Medicine Use & 77 & 69 & 46 & $* *$ \\
\hline Mood Stabilizers Medicine Use & 23 & 8 & 8 & $* * *$ \\
\hline Anti-Psychosis Medicine Use & 54 & 31 & 15 & $* * *$ \\
\hline \multicolumn{5}{|l|}{ CAQ } \\
\hline Self-Harming & 69 & 38 & 8 & $* * * *$ \\
\hline Suicide Attempts & 31 & 15 & 8 & $* * * *$ \\
\hline Temper Tantrum And Social Fights & 92 & 54 & 46 & $* * *$ \\
\hline Drug And Medicine Abuse & 54 & 30 & 15 & $* *$ \\
\hline Legal Problems & 15 & 0 & 0 & $* * * *$ \\
\hline Urgent Hospital Recovery & 53 & 0 & 0 & $* * * *$ \\
\hline Use Of Medicine In The Last Year & 100 & 92 & 0 & $* * * *$ \\
\hline Use Of Medicine Now & 93 & 69 & 30 & $* * *$ \\
\hline Urgent Treatment & 92 & 61 & 39 & $* * *$ \\
\hline No Work Or Precariat & 46 & 15 & 7 & $* * * *$ \\
\hline Not Autonomous Living & 46 & 38 & 31 & $* *$ \\
\hline
\end{tabular}

*low change: delta $25 \%, * *$ medium low change: delta $26-50 \%, * * *$ medium high change: delta $51-75 \%, * * * *$ high change: delta $>75 \%$. 
while $50 \%$ were employers; all of them were unmarried and just $50 \%$ were high school graduated. About the females, $75 \%$ of them have a job, while $25 \%$ were unoccupied; $75 \%$ of them were unmarried and just $25 \%$ of them were engaged, and were high school graduated, none of them has a degree. Their average Socio-Economical Status (Hollingshead, 1975) of the group was medium-low.

\section{Procedure}

The micro-analytic research protocol was provided in the following steps: i)

i) Time $0\left(\mathrm{~T}_{0}\right)$ : pre-treatment screening

ii) Time $3 \mathrm{~m}: 3$ months from the beginning of the project.

iii) Time 6m: 6 months from the beginning of the project.

iv) Time $1\left(\mathrm{~T}_{1}\right): 9$ months from the beginning of the project.

The project is still ongoing: so, we here we will consider only the preliminary results of the micro-analytic path of mentalization changes.

\section{Measures}

As for the previous study, they are both self- and clinician-report tools. In this second study, all the tools are administered by the clinician and self-administered by the patient at the end of the session to which they refer: each clinician gives an evaluation about his/her single patient in a specific psychotherapy session. These tools are administered in a session every 3 months.

RFQ (Fonagy et al., 2016) is a self-report designed to allow an agile measurement of mentalization capacity, or reflexive function. It consists of 8 items on 7-point Likert scale and lower marks corresponded to better mentalization skills. It presents 2 factors: Certain Mentalization, and Uncertain Mentalization. It was administered just at $\mathrm{T}_{0}$ and $\mathrm{T} 1$.

Modes of Mentalization Scale (MMS) is a clinicianreport questionnaire, which permits the clinician to evaluate the patient's modalities of mentalization (teleological attitude, psychic equivalence, pseudo-mentalization and good mentalization). It consists of 32 items, for each of which the clinician appraises, taking into account both the contents and the narratives of the patient, and the way he relates to the clinician, on a 6-point Likert scale $(0=\mathrm{Ab}$ solutely non-descriptive; $5=$ Absolutely descriptive).

Mentalization Imbalances Scale (MIS) (Gagliardini \& Colli, 2018) is a clinician-report questionnaire that permits the clinician to evaluate the patient's imbalances of mentalization (Self/Other, Internal/External, Affective/ Cognitive, Implicit/Explicit). It consists of 49 items, for each of which the clinician appraises, taking into account both the contents and the narratives of the patient, and the way he relates to the clinician, on a 6-point Likert scale $(0=$ Absolutely non-descriptive; $5=$ Absolutely descriptive). Validation studies are ongoing (Gagliardini, Caverzasi, Boldrini, Ferrero, \& Colli, 2018; Gagliardini et al., 2017).
Comparative Psychotherapy Process Scale (CPPS) (Hilsenroth, Blagys, \& Ackerman, 2005) is a self-assessment scale with which the clinician assesses the characteristics and complexity of his/her interventions. It consists of 20 items on the 7-point Likert scale $(0=$ Not at all characteristic, $5=$ Extremely characteristic). While the scale may be used for an a-theoretical evaluation of psychotherapy process, it should be noted that the CPPS items are derived from empirical studies comparing/contrasting Psychodynamic-Interpersonal (PI) and CognitiveBehavioral (CB) approaches to treatment.

Session Evaluation Questionnaire - Patient version and Clinician version (SEQ-P and SEQ-T) (Rocco, Salcuni, \& Antonelli, 2017) is a self-report questionnaire prepared in double version (for the clinician and for the patient), gathering a multiple-informants perspective along with a 7-degree semantic differential scale. The patient version consists of 27 items about the just-ended session: 12 of them concern the patient's perception/sensations about the session; 12 of them interrogate the patient about his/her personal experience; 3 of them investigate the patient's perception of the therapist. The structure of the therapist version is identical, but it counts 24 items because the 3 items relating to the therapist's perception are missing. The double version allows of the comparison of patient's $v s$ therapist's session evaluation in terms of concordance/discrepancy. Moreover, it is possible to evaluate the trend of single item or dimension over time.

\section{Data analysis}

The micro-analytical changes of a new group of 6 patients was analyzed with Friedman test, a non-parametric test to compare at $\mathrm{T}_{0}, 3,6$ and 9 months $\left(\mathrm{T}_{1}\right)$ mentalization and session evaluation trends (SEQ, CPPS, MMS, MIS), as well as the reflective functioning at $\mathrm{T}_{0}$ and $\mathrm{T}_{1}$ (RFQ).

\section{Results}

The administration of self-report (SEQ-P, SEQ-Q, CPPS) and clinician-report (MMS, MIS) tools enabled to assess mentalization by adopting a multidimensional perspective. Only some dimensions are statistically significant, but we can make some clinical reflections.

Looking at Table 4, it is possible to notice a decrease of the arousal in therapists during the initial 9 months of treatment: the high level of anxiety of the therapist at the beginning of the treatment may be due to the high relational distress that these patients manifest, and we could suppose an habituation or familiarity process during the unfolding of the therapy. However, none of the different dimensions measured with the SEQ show statistically significant changes in the first 9 months of treatment.

As Table 5 shows, therapists' interventions enlightened an increase of a psychodynamic dimension over time, while cognitive-behavioral perspective weren't related to a statistically significant change. 
By Table 6, it is possible to observe the constant decrease with respect to the use of hyper-mentalization, concrete comprehension and teleological mentalization in favor of a statically significant improvement in the use of good mentalization.

Moreover, about the MIS, between $\mathrm{T}_{0}$ and $\mathrm{T}_{1}$ it is possible to notice a reduction in the unbalancing of all polarities. The decrease in Cognitive unbalance, Automatic unbalance and Self-unbalance is statistically significant, which means an improvement in mentalizing skills. RFQ rated at $T_{0}$ and $T_{1}$ enlightened a significant improvement of the Certain Mentalization $(\mathrm{M}=1.08, \mathrm{sd}=1.53$, and $\mathrm{M}=.33$, $\mathrm{sd}=.47$, respectively), and conversely an impoverishment of Uncertain Mentalization $(\mathrm{M}=.83, \mathrm{sd}=1.18$ and $\mathrm{M}=1.92, \mathrm{sd}=.59$, respectively).

\section{Discussion and Conclusions}

In the first study, our research hypothesis was to test if it was possible to apply an oriented MBT care path in an Italian Public Health Service and to verify if it was associated with a improvement of patients' symptomatology, emotional regulation (above all of self- and hetero-directed rage and aggression, linked to self-harming, temper tantrum, social fights and suicidal behaviors), prevention and management of self-injurious and anticonservative behaviors, interpersonal relationships and with a reduction of the workload for the Service staff and of the costs of assistance. The initial results of the longitudinal oriented MBT projects on a 9 patients group compared at the beginning of the treatment $\left(\mathrm{T}_{0}\right)$, at the end of

Table 4. Comparison for non-parametrical repeated measures at the beginning $\left(\mathrm{T}_{0}\right)$, after 3,6 and 9 months $(\mathrm{N}=6$ patients) at Session Evaluation Questionnaire-Patient (SEQ-P) and Session Evaluation Questionnaire-Therapist (SEQ-T).

\begin{tabular}{|c|c|c|c|c|c|c|c|c|c|c|}
\hline & & & & $3 \mathrm{mo}$ & & $6 \mathrm{mon}$ & & $\mathrm{T}_{1}-9 \mathrm{mc}$ & ths & Friedman Test \\
\hline & & Mean & sd & Mean & sd & Mean & sd & Mean & sd & $* P<.05$ \\
\hline SEQ-P & Depth & 2,9 & 0,8 & 2,9 & 0,7 & 2,1 & 0,4 & 2,1 & 0,6 & n.s \\
\hline & Smooth & 3,4 & 1,2 & 3,9 & 1,4 & 3,8 & 2,0 & 3,3 & 1,2 & n.s \\
\hline & Positivity & 4,0 & 0,7 & 3,6 & 1,1 & 4,1 & 0,7 & 3,9 & 0,9 & $n . s$ \\
\hline & Arousal & 4,1 & 0,9 & 3,8 & 0,7 & 3,3 & 1,1 & 3,3 & 1,2 & n.s \\
\hline & Therapist & 4,8 & 0,8 & 4,7 & 0,4 & 4,4 & 0,6 & 4,2 & 1,3 & $n . s$ \\
\hline SEQ-T & Depth & 3,3 & 1,1 & 3,0 & 0,6 & 2,8 & 1,0 & 2,4 & 0,4 & n.s \\
\hline & Smooth & 3,4 & 1,5 & 3,7 & 1,7 & 3,8 & 1,0 & 3,5 & 1,4 & n.s \\
\hline & Positivity & 2,9 & 0,6 & 2,9 & 0,3 & 2,8 & 0,5 & 2,8 & 0,3 & n.s \\
\hline & Arousal & 4,1 & 0,8 & 3,8 & 0,8 & 3,1 & 0,6 & 3,0 & 0,6 & $*$ \\
\hline
\end{tabular}

Table 5. Comparison of non-parametrical repeated measures at the beginning $\left(T_{0}\right)$, after 3,6 and 9 months $(\mathrm{N}=6$ patients) at Comparative Psychotherapy Process Scale (CPPS).

\begin{tabular}{|c|c|c|c|c|c|c|c|c|c|c|}
\hline \multirow{3}{*}{ CPPS } & \multirow{3}{*}{$\begin{array}{l}\text { Psychodynamic-Interpersonal } \\
\text { Cognitive-Behavioral }\end{array}$} & \multirow{2}{*}{\multicolumn{2}{|c|}{ 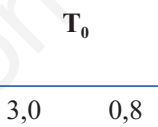 }} & \multicolumn{2}{|c|}{3 months } & \multicolumn{2}{|c|}{6 months } & \multicolumn{2}{|c|}{$\mathrm{T}_{1}-9 \mathrm{months}$} & \multirow{2}{*}{$\begin{array}{c}\begin{array}{c}\text { Friedman Test } \\
* \mathbf{P}<.05\end{array} \\
*\end{array}$} \\
\hline & & & & 2,8 & 0,6 & 3,0 & 0,6 & 3,2 & 0,5 & \\
\hline & & 1,3 & 0,9 & 1,9 & 0,5 & 1,3 & 0,8 & 1,2 & 0,4 & $n . s$ \\
\hline
\end{tabular}

Table 6. Comparison of non-parametrical repeated measures at the beginning $\left(\mathrm{T}_{0}\right)$, after 3,6 and 9 months $(\mathrm{N}=6$ patients) at Modes of Mentalization Scale (MMS) and Mentalization Imbalance Scale (MIS).

\begin{tabular}{|c|c|c|c|c|c|c|c|c|c|c|}
\hline \multirow[b]{2}{*}{ MMS } & \multirow[b]{2}{*}{ Hyper-mentalization } & \multicolumn{2}{|c|}{$\mathbf{T}_{\mathbf{0}}$} & \multicolumn{2}{|c|}{3 months } & \multicolumn{2}{|c|}{6 months } & \multicolumn{2}{|c|}{$\begin{array}{l}\mathbf{T}_{1}-9 \text { months } \\
* \mathbf{P}<.05\end{array}$} & \multirow{2}{*}{$\begin{array}{c}\text { Friedman Test } \\
n . s\end{array}$} \\
\hline & & 3,2 & 1,3 & 2,6 & 1,2 & 2,9 & 0,8 & 2,8 & 0,9 & \\
\hline & Concrete Comprehension & 2,7 & 0,6 & 2,9 & 1,0 & 2,8 & 0,5 & 2,5 & 0,4 & n.s \\
\hline & Good Mentalization & 2,0 & 0,7 & 2,2 & 0,5 & 2,6 & 0,6 & 2,8 & 0,8 & $*$ \\
\hline & Teleological mentalization & 3,5 & 1,1 & 3,2 & 1,2 & 3,4 & 0,7 & 2,7 & 1,0 & n.s \\
\hline & Pseudo Intrusive Mentalization & 2,5 & 0,7 & 2,1 & 0,8 & 2,2 & 0,7 & 2,4 & 0,9 & $n . s$ \\
\hline \multirow[t]{5}{*}{ MIS } & Cognitive Imbalance & 3,3 & 0,8 & 2,8 & 1,4 & 2,8 & 1,3 & 2,5 & 1,5 & $*$ \\
\hline & Affective Imbalance & 3,6 & 0,9 & 3,2 & 0,9 & 3,3 & 0,9 & 3,1 & 0,9 & n.s \\
\hline & Other-Imbalance & 3,1 & 0,9 & 2,5 & 0,7 & 2,5 & 0,9 & 2,6 & 0,8 & n.s \\
\hline & Automatic Imbalance & 3,4 & 0,8 & 3,0 & 0,6 & 3,3 & 0,7 & 2,4 & 0,8 & $*$ \\
\hline & Self-Imbalance & 3,5 & 0,8 & 3,3 & 0,7 & 3,3 & 0,5 & 2,8 & 0,6 & $*$ \\
\hline
\end{tabular}


the treatment $\left(\mathrm{T}_{2}\right)$ and 1 year (FU1) after the end of the care path, showed a statistical and clinical reduction of self-reported symptoms (SCL-90R); moreover, clinicians and psychodiagnostic scales about global health functioning (HoNOS, SCID-II, GAF) were related to improvements. We also found benefits in terms of service impact and service costs (PES and folder data).

Our results, in line with the theory of Bateman and Fonagy (1999), showed that the group of patients participating to the project had important changes from the first months of treatment, with respect to suicide attempts, to self-harm behaviors and to the frequency and duration of psychiatric admissions. The improvements obtained in these areas increased during the following months and were maintained during the follow-up at 18 months after the conclusion of the path (Bateman \& Fonagy, 2001, 2008a, 2008b).

Bateman and Fonagy (2013a, 2013b) affirm that the subjects with a good mentalizing capacity can oscillate in a balanced and flexible way between the polarities of mentalization, depending on the situations and the relational contexts, while the people with BPD show a more rigid pattern of functioning, with an imbalance between the polarities. The second study was looking for changes in patients' mentalization ability during an oriented MBT care path (in terms of imbalance and modes of mentalizing, in order to see if these changes could be accompanied by differences in other processual dimensions. The data trend, even in such a brief period, seems to indicate an improvement of mentalizing skills during the oriented MBT path. The analysis of a new group of patients who began the MBT protocol recently showed a reduction of these imbalances in favor of a more stable balance between the polarities correlated with the increment of mentalizing capacity. These results are based on to the first 9 months of treatment, and we may hypothesize an increase in this improvement over the next 9 months. Our results enlighten the clinical effectiveness of MBT, and the increase in mentalization capacity following the first months of treatment, confirming Bales et al.'s results (2012). This study suggested that changes in patients' mentalization capacity and in its modes and polarities were accompanied by a higher presence of therapist's psychodynamic-interpersonal interventions and by a reduction of therapists' arousal during the course of treatment.

Our paper verified that it was possible to implement an oriented MBT care path, as articulated by Fonagy and Bateman, in an Italian Public Service. This was the first important attempt of MBT in Italy in a Public Service; it was substantially based on the voluntary will of the medical and psychological staff, not funded by Italian Health Care National Service. The first results proved, in terms of cost effectiveness and clinical healing, how it is important to increase prevention and to develop organized therapeutic interventions, in order to contain the impact of urgent interventions. This result had a direct effect, in terms of a reduction of workload for the Service staff and of the costs of assistance.

As expected, despite the insane level of impairment in relation to the personality pathology of the patients, oriented MBT intense protocol was able to improve patients' overall level of functioning, to reduce the presence of symptoms typically associated with $\mathrm{BPD}$, and to improve both relational skills and diagnostic criteria for BPD in participants after an 18 months treatment. This change remained quite stable at the follow-up after one-year, both from the patient's self-perspective and the clinicians' one. Considering the microanalytic change, patients' mentalization capacity seemed to increase in respect with a better and authentic mentalization rather than a pseudologic one.

Despite the promising results, this paper showed several important limitations. First of all, the preliminary data involved just a small group of patients that had finished the program. Since 2014, 27 patients have been treated with MBT; 13 of them have already completed the path, while 8 are still under treatment; just 9 patients of the 13 completed the path the one-year follow-up. At the beginning, 6 recruited patients (22\%) dropped along the first 3 months (so they were excluded from the present study): one of them has abandoned the treatment due to a transfer of residence, one due to an incompatibility of the group sessions with their working hours, while the remaining dropped-out because of a personal difficulty in tolerating the stimuli deriving mainly from the group setting.

A second important limitation is that we adapted the original Bateman and Fonagy (2010a) MBT program for the Camposampiero MHC setting, so we might state we use a oriented $M B T$ protocol which adherence to the original one was not precisely measured.

In addition, the study is based only on self-report and clinician-report tests, and a particularly tricky fact with our patients is they seem to be better functioning than patients that are usually included in other MBT programs. In the result section, we report that mean BPD criterion is 5 , but the functional level is higher than what is ordinarily found in MBT cohorts. Mean GAF at admission is here 54 and after 18 months it is 75 and at FU it is 77. Roughly, this is around $10 \mathrm{GAF}$ point higher than in other studies (Bateman, O'Connell, Lorenzini, Gardner \& Fonagy, 2016). A question we might answer is since GSI is a self-report, could we admit that this Italian cohort is a group of patients that are functioning on a level which is significantly higher than in other studies? If this is true, then we know that in some other countries such patients would not have been admitted to an MBT program which is designed for poorly functioning patients. It could be that (involuntary) we chose to begin this work with patients who were functioning better in order to train our staff and the system and thereafter extend it to more disturbed patients.

Finally, in line with our preliminary results and with the findings from Bateman and Fonagy (2008a, 2008b), 
we qualitatively showed that, despite the substantial investment required by a partial hospitalization regime, dayhospital treatment may lead to an increased reduction in sanitary costs after the end of treatment, when compared to the TAU performed as an outpatient procedure; however, the quantitative reduction of costs was not yet provided for the considered period neither for prolonged follow up evaluations.

The extension of the sample size and the inclusion of more severe patients will allow the clarification of the dynamics underlying the improvements found in the present study and to provide further data for more accurate statistical analyses, which should not include only non-parametric statistics. As a future direction adherence and competence tools might be administered - such as Adherence and Competence Scale of the Mentalization-Based Treatment (MBT-ACS) (Karterud et al., 2012) - as well as projective or narrative measures behind questionnaires.

Moreover, although the intensive outpatient MBT treatment is challenging for both patients and the équipe, it would be interesting to check, as Bateman and Fonagy did (2003, 2008a, 2008b), whether the costs of hospital treatment are canceled by the reduction of expenses due to hospitalization in psychiatric wards or emergency. For the future, it could be tested whether the decrease in terms of use of Health Services is related to an effective monetary reduction of costs.

The authors (Bateman \& Fonagy, 2008a, 2008b) concluded that patients treated with MBT, in addition to maintaining the benefits they had gained over the 18 months, also showed a continuous improvement of all dimensions considered, suggesting that MBT had induced a process of long-term change. It would therefore be very interesting to be able to assess whether the benefits obtained with the treatment have persisted not only after one year from the end of the project (FU1), but also in the following years.

In conclusion, standing all the limits about adherence to standard MBT, little sample size, and puzzling data, and lack of a quantitative cost-effectiveness measurement, an important merit of this paper is that it is the first clinical report of an oriented MBT program in Italy and we hope it would have the potential to stimulate a fruitful clinical discussion.

\section{References}

American Psychiatric Association - APA (2000). Manuale diagnostico e statistico dei disturbi mentali (4th ed). Testo riveduto (DSM-IV-TR). Washington, DC: American Psychiatric Publishing.

American Psychiatric Association - APA (2005). EvidenceBased Practice in Psychology. American Psychologist, 61(4), 271-285.

American Psychiatric Association - APA (2013). Manuale diagnostico e statistico dei disturbi mentali - 5th ed. (DSM-V). Washington, DC: American Psychiatric Publishing.
Bales, D., van Beek, N., Simts, M., Willemsen, S., Busschbach, J. J. V., Verheul, R., \& Andrea, H. (2012). Treatment outcome of 18-month day hospital Mentalization-Based Treatment (MBT) in patients with severe borderline personality disorder in the Nederlands. Journal of Personality Disorders, 26(4), 568-582.

Bateman, A. W. \& Fonagy, P. (1999). Effectiveness of partial hospitalization in the treatment of borderline personality disorder: a randomized controlled trial. American Journal of Psychiatry, 156, 1563-1569.

Bateman, A. W. \& Fonagy, P. (2001). Treatment of borderline personality disorder with psychoanalytically oriented partial hospitalization: a 18 month follow-up. American Journal of Psychiatry, 158, 36-42.

Bateman, A. W. \& Fonagy, P. (2003). Health service utilization costs for borderline personality disorder patients treated with psychoanalytically oriented partial hospitalization versus general psychiatric care. American Journal of Psychiatry, 160, 169-171.

Bateman, A. W. \& Fonagy, P. (2008a). 8-Year follow-up of patients treated for borderline personality disorder: mentalization-based treatment versus treatment as usual. American Journal of Psychiatry, 165, 631-638.

Bateman, A. W. \& Fonagy, P. (2008b). Randomized controlled trial of outpatient mentalization-based treatment versus structured clinical management for borderline personality disorder. American Journal of Psychiatry, 166, 1355-1364.

Bateman, A. W. \& Fonagy, P. (2010a). Guida pratica al Trattamento Basato sulla Mentalizzazione per il disturbo borderline di personalità. Varese, Italy; Raffaello Cortina Editore.

Bateman, A. W. \& Fonagy, P. (2010b). Mentalization based treatment for borderline personality disorder. World Psychiatry, 9, 11-15.

Bateman, A. W. \& Fonagy, P. (2013a). Handbook of Mentalizing in Mental Health Practice. Washington, DC: American Psychiatric Publishing.

Bateman, A. W. \& Fonagy, P. (2013b). Impact of clinical severity on outcomes of mentalisation-based treatment for borderline personality disorder. The British Journal of Psychiatry, 203, 221-227.

Bateman, A., O'Connell, J., Lorenzini, N., Gardner, T., Fonagy, P. (2016). A randomized controlled trial of mentalizationbased treatment versus structured clinical management for patients with comorbid borderline personality disorder and antisocial personality disorder. BMC Psychiatry, 16(1), 304.

Bergonzini, C., Raffaelli, S., Prelati, M., Piantato, E., Bertamini, D. \& Veglia, F. (2013). Studio preliminare su un campione di soggetti con diagnosi di disturbo di personalità borderline ricoverati presso il Day Hospital Psichiatrico della Struttura di Psichiatria S.P.D.C. dell'Azienda Ospedaliera "SS. Antonio e Biagio e Cesare Arrigo" di Alessandria. Working Paper of Public Health, 2, 1-13.

Chapman, J., \& Fleisher C. (2017). Personality disorder, borderline. Available from: https://www.ncbi.nlm.nih.gov/books/ NBK430883/.

David, D., Lynn, S. J., \& Montgomery, G. (2018). Evidencebased psychotherapy: the state of the science and practice. Hoboken, NJ: John Wiley \& Sons, Inc.

Derogatis, L.R. (1994). Symptom checklist-90-R: administration, scoring, and procedures manual (3rd ed.). Minneapolis, MN: National Computer Systems.

First, M. B. \& Gibbon, M. (2004). The Structured Clinical Interview for DSM-IV Axis I Disorders (SCID-I) and the 
Structured Clinical Interview for DSM-IV Axis II Disorders (SCID-II). In M. J. Hilsenroth \& D. L. Segal (Eds.), Comprehensive handbook of psychological assessment, Vol. 2. Personality assessment (pp. 134-143). Hoboken, NJ, US: John Wiley \& Sons Inc.

Fonagy, P. (2000). Attachment and borderline personality disorder. Journal of American Psychoanalytical Association,48, 1129-1146.

Fonagy, P., \& Allison, E. (2011). What is mentalization? the concept and its foundations in developmental, in N. Midgley \& I. Vrouva (Eds). Keeping Children in Mind: Mentalizationbased Interventions with Children, Young People and their Families. New York, NY: Sage.

Fonagy, P., Bateman, A., \& Luyten, P. (2012). Introduction and overview. In A. Bateman \& P. Fonagy (Eds.). Handbook of mentalizing in mental health practice (pp. 3-41). Arlington, VA: American Psychiatric Publishing.

Fonagy, P., Gergely, G., \& Target, M. (2007). The parent-infant dyad and the construction of the subjective self. Journal of Child Psychology and Psychiatry, 48, 288-328.

Fonagy, P., Luyten, P., Moulton-Perkins, A., Lee, Y.W., Warren, F., Howard, S., ... Lowyck, B. (2016). Development and validation of a self-report measure of mentalizing: the reflective functioning questionnaire. PLoS ONE, 11(7), e0158678.

Fonagy, P., Steele, M., Steele, H., Moran, G.S., \& Higgit, A.C. (1991). The capacity of understanding mental states: The reflective self in parent and child and its significance for security of attachment. Journal of Consulting and Clinical Psychology, 74, 1027-1040.

Fonagy, P., Target, M., \& Bateman, A. (2018). The mentalization based approach to psychotherapy for borderline personality disorder. In P. Williams, ed. The Psychoanalytic Therapy of Severe Disturbance. London, UK: Routledge.

Gabbard, G.O. (2015). Psichiatria psicodinamica - Quinta Edizione basata sul DSM-5. Milan, Italy: Raffaello Cortina Editore.

Gagliardini, G., \& Colli, A. (2018). Assessing mentalization: development and preliminary validation of the modes of Mentalization Scale. Psychoanalytic Psychology. doi: 10.1037/pap0000222.

Gagliardini, G., Caverzasi, E., Boldrini, A., Ferrero, A., \& Colli, A. (2018). Assessing mentalization with the Mentalization Imbalances Scale. Paper presented at the 20th Conference of the Italian Association of Psychology - Clinical and Dynamic Psychology Section, Urbino, Italy. Mediterranean Journal of Clinical Psychology, 6(2), 109.

Gagliardini, G., Caverzasi, E., Boldrini, A., Ferrero, A. Blasi, S., Gullo, S., \& Colli, A. (2017). Development and first validation of a new clinician report to assess mentalization: the modes of Mentalization Scale. Paper presented at the Research About Mentalizing Workshop, 2017, Milan, Italy.

Gunderson, J.G. \& Links, W.P. (2014). Handbook of good psychiatric management for borderline patients. Washington, DC: American Psychiatric Publishing.

Hilsenroth, M. J., Blagys, M. J., \& Ackerman, S. J. (2005).
Measuring psychodynamic-interpersonal and cognitive-behavioral techniques: development of the comparative psychotherapy process scale. Psychotherapy: Theory, Research, Practice, 42(3), 340-356.

Hollingshead, A.B. (1975). Four factors index of social status. New Haven: Yale University Department of Sociology.

Jones, S.H., Thornicroft G., Coffey M. \& Dunn, G (1995). A brief mental health outcome scale-reliability and validity of the Global Assessment of Functioning (GAF). The British Journal of Psychiatry, 166, 654-659.

Jorgensen, K.R. \& Kjolbye, M. (2007). Outcome of psychoanalytically outpatient tratment of borderline personality disorder: a pilot study. Nord Psykol, 59, 164-180.

Karterud S., Pedersen G., Engen M., Johansen MS, Johansson PN, Schlüter C, ... Bateman AW. (2012). The MBT Adherence and Competence Scale (MBT-ACS): development, structure and reliability. Psychotherapy Research, 23, 705-17.

Levy, K.N., Meehan, K.B. \& Kelly, K.M. (2006). Change in attachment and reflective function in the tratment of borderline personality disorder with tranference-focused psychotherapy. Journal of Consulting and Clinical Psychology, 74, 1027-1040.

Lora, A. (2009). An overview of the mental health system in Italy. Annali dell'Istituto Superiore di Sanità, 45, 5-16.

Lora, A., Bai, G., Bianchi, S., Bolongaro, G., Civenti, G., Erlicher, A., ... \& Morosini, P. (2001). The Italian version of HoNOS (Health of the Nation Outcome Scales), a scale for evaluating the outcomes and the severity in mental health services (pp. 198-204). Cambridge: Cambridge University Press.

Madeddu, F., Aquaro \& P., Preti, E. (2012). Psicoterapia per il disturbo borderline di personalità: revisione della letteratura sull'efficacia di quattro trattamenti manualizzati. Journal of Psychopathology, 18, 196-209.

Morandotti, N., Brondino, N., Merelli, A., Boldrini, A., De Vidovich, GZ., \& Ricciardo, S. (2018). The Italian version of the Reflective Functioning Questionnaire: Validity data for adults and its association with severity of borderline personality disorder. PLoS One, 13(11), e0206433.

Rocco, D., Salcuni, S. \& Antonelli, E. (2017). A pilot study of the Italian adaptation of the Session Evaluation Questionnaire fourth version. Research in Psychotherapy: Psychopathology, Process and Outcome, 20(2), 122-130.

Sanza, M., Asioli, F. \& Ferrannini, L. (2010). Disturbo Borderline di Personalità: Continuità e discontinuità nel trattamento. Milan, Italy: Edi.Ermes.

Slade, A., Grienenberger, J., Bernbach, E., Levy, D. \& Locker, A. (2005). Maternal reflective functioning, attachment, and the trasmission gap: A preliminary study. Attachment and Human Development, 7, 283-298.

Torgensen, S., Kringlen, E. \& Cramer, V. (2001). The prevalence of personality disorders in a community sample. Archives of General Psychiatry, 58, 590-596.

Wing, J.K., Beevor, A.S., Curtis, R.H., Park, S.B., Hadden, S. \& Burns, A (1998). Health of the Nation Outcome Scales (HoNOS). Research and development. The British Journal of Psychiatry, 172, 11-18. 